\title{
Collective Effort on Restriction Enzymes
}

BACTERIA are able to recognize and destroy foreign DNA, and this defence mechanism is the subject of several current investigations. A further contribution in this field has come simultaneously from laboratories in San Francisco (see page 40 of this issue) and Edinburgh (Nature New Biology, 244, 7 ; 1973). Both Boyer et al. and Bigger et al. have determined the sites of attack and at least a part of the nucleotide sequence of the sites in DNA recognized by the Escherichia coli RII restriction enzyme. Boyer et al. have shown, furthermore, that the recognition site for the corresponding methylating enzyme includes the same pentanucleotide sequence on the DNA which is recognized by the restriction enzyme. The source of DNA is immaterial: the vulnerable sequences are the same in SV40 (an animal viral) DNA and $\lambda$ phage DNA.

It is known that restriction and modification of DNA in bacteria go hand in hand. The genetic loci which determine these activities generally are closely linked and, in some cases, it seems that the same protein performs both functions. The function of restriction enzymes (specific deoxyribonucleases) is to nick invading DNA. Homologous cellular DNA is protected because it is modified (by methylating enzymes) in the target sites of the restriction enzymes. Modification renders the host DNA resistant to attack. Moreover, restriction enzymes require double stranded DNA, and modification of either of the two strands (for example, the parental strand of replicating DNA) is sufficient to discourage the enzyme. This ingenious mechanism ensures that nascent DNA of the host cell is safe from attack. Apparently, once the invading DNA is "nicked", a number of other deoxyribonucleases degrade it even further and prevent its repair by enzymes (ligases) known to exist in bacteria.

The work of Boyer et al. and Bigger et al. shows that the $E$. coli $\mathrm{RII}$ restriction enzyme prefers the pentanucleotide sequence :

$$
\begin{aligned}
& \ldots \text { N-C-C-A-G-G-N } \ldots \\
& \ldots \text { N-G-G-T-C-C-N } \ldots
\end{aligned}
$$

(representing the antiparallel strands $5^{\prime}-3^{\prime}$ at the top and $3^{\prime}-5^{\prime}$ at the bottom of the sequence). In both strands phosphodiester cleavage is at the $5^{\prime}$ cytidylic acid residue, and the products have frayed ends terminating in a phosphorylated 5' C "tail" and a 3'-OH nucleoside on the short leg. The evidence for this model comes from conventional sequence studies (thanks in part to some of the authors represented here), from the demonstration by electron microscopy that the products have cohesive termini, and from sequence studies on the DNA repaired with RSV DNA polymerase and labelled nucleotides. It is, incidentally, the Cs that are methylated in the pentanucleotide sequence of DNA immune to the RII restriction enzyme.

Bigger $e t$ al. note that such a pentanucleotide sequence would be expected to occur randomly about forty-five times in $\lambda$ DNA, which contains 46,500 base pairs. They demonstrate that about this number of breaks (in fact twenty) actually occur, judging from the number of fragments yielded by the purified restriction enzyme acting in vitro. It is therefore interesting to speculate that the restriction enzymes have evolved in response to a need for selecting out sequences of a particular character which occur in DNA by chance.

Comparing the sequences attacked by restriction enzymes of different specificity obtained from various strains of bacteria has led to a general theory concerning the nature of the likely targets and the mechanism of action of these enzymes. The restriction enzyme from Haemophilus influenzae, for example, favours the hexanucleotide sequence :

$$
\begin{aligned}
& \text {.. N-G-T-Py-Pu-A-C-N . . . } \\
& \ldots \text { N-C-A-Pu-Py-T-G-N . . }
\end{aligned}
$$

(written according to the convention stated earlier). Other restriction nucleases leave longer or shorter tails, and the sequences recognized and positions of the phosphodiester cleavages vary. One common denominator, however, is a dyad axis of symmetry down the centre of the sequence. In the case of the RII restriction enzyme, the dyad axis passes perpendicularly down through the middle of the AT pair of the pentanucleotide sequence; in the Haemophilus influenzae restriction enzyme it passes between the purine and pyrimidine residues in the complementary strands of the relevant hexanucleotide sequence.

The enzyme may thus act by loosening the double helix in the vulnerable sequence causing the formation of two rotationally symmetrical hairpin loops on opposite strands. These loops may be envisaged to fit nicely into the active site(s) of the enzyme molecule which bites the stem at specific points. Kinetic studies suggest that one of the two nicks occurs a few seconds after the other, so that digestion is not necessarily coordinated in time. In order to act in this fashion it is suggested that restriction enzymes may have (at least) two identical subunits. Some of the restriction enzymes which have been purified and studied do indeed conform to this expectation, at least structurally. The variation, however, reflected not least in the wide range of molecular weights discovered for these enzymes-70,000-450,000 to date-promises to be a fascinating subject for protein physical chemists in the future.

The byproducts of this work may be no less interesting than the findings themselves. It may be noted that many proteins besides nucleases and modification enzymes must recognize specific sequences on nucleic acids: repressors, polymerases, ribosomal proteins and chromosomal proteins are but a few. The consequences of a mutation affecting the function of a modification enzyme, unlike some of the others, .however, may end in suicide. It may be anticipated therefore that the nucleoprotein interactions must exhibit an unusually high degree of specificity.

A second very useful byproduct of the collective effort on restriction enzymes will, of course, be the provision of a variety of specific hydrolases for degrading DNA in a specific manner. This may finally open up the difficult field of DNA sequencing. The discovery that partial digestion of RNA (for example, with pancreatic and T1 ribonuclease) occurs in a specific fashion made the field of RNA sequencing blossom out a few years ago, yielding a splendid variety of results.

H. G. 